\title{
INVESTIGATING THE REMOTE MONITORING USAGE FOR HOME HEALTH CARE
}

\author{
Type of article: conference abstract
}

\author{
Parinaz Tabari1*, Najme Montaseri1 \\ 1: Department of Health Information Management, School of Allied Medical Sciences, \\ Tehran University of Medical Sciences, Tehran, Iran \\ *p-tabari@razi.tums.ac.ir, papiamentu@gmail.com
}

\begin{abstract}
Introduction: Managing and monitoring some diseases and disorders are costly and complicated for patients, their families and healthcare systems. Furthermore, many of the pharmaceutical facilities, equipment and disease management programs are not available to all people in the community. Therefore the use of wireless technologies along with the telephone-based transmission systems, can collect the patients' health status data and share with related providers. Remote monitoring defines a management approach using communication technology to track patients' health.

Methods: In this study, we searched recent articles indexed in PubMed, Science Direct, Ovid, Web of Science and Google Scholar to investigate articles which aimed to explore the use of remote patient monitoring systems in different clinical status and to review the advantages and disadvantages of these systems.

Results: People with chronic diseases such as heart disease have complicated care needs. If these people would not be exposed to proper interventions, this situation may lead to multiple referral to the emergency departments and even re-hospitalization. Home care by using clear communication protocols, can have a significant impact on improving the quality of care and safety of patients after discharging from the hospital. In such chronic diseases, remote monitoring fascilities and interventions along with education can reduce the use of healthcare resources and the need for early re-admission. Remote monitoring can also support people with disturbances of consciousness. In the field of drug prescription, this technology can also offer recommendations for regulation and alteration of last prescribed drugs in addition to suggestions on patient behavioural changes by care providers and predefined algorithms. This technological method can also be used to monitor side effects of some medications such as antihypertensive drugs.

Conclusion: Monitoring patients remotely, is most commonly used in heart diseases, pulmonary diseases, diabetes and blood pressure diseases. Studies have also been conducted in areas such as serving the elderly and drug counseling. Although there are contradictions about the impact and effectiveness of this approach on some diseases, for patients who are hospitalized frequently, a daily program for monitoring them remotely can have an impact on optimizing health care resource utilization and reduce the number of admissions and length of stay in the hospital and ultimately improve the quality of life of the individual.
\end{abstract}

KEYWORDS: Remote monitoring, self management, chronic disease.

\section{Declaration of conflicts}

This abstract is selected from the First International Congress of Diseases and Health Outcomes Registry and First National Congress of Medical Informatics, 14-17 February 2017, Mashhad, Iran

\section{Authors' biography}

No biography.

\section{References}

No references. 